\title{
The use of intraoperative navigation for complex upper cervical spine surgery
}

\author{
Report of 4 cases
}

\author{
Kern H. Guppy, M.D., Ph.D., Indro Chakrabarti, M.D., M.P.H., \\ AND Amit BanerJeE, M.D. \\ Department of Neurosurgery, Kaiser Permanente Medical Group, Sacramento, California; and Department \\ of Neurosurgery, University of California, San Francisco, California
}

\begin{abstract}
Imaging guidance using intraoperative $\mathrm{CT}$ ( $\mathrm{O}$-arm surgical imaging system) combined with a navigation system has been shown to increase accuracy in the placement of spinal instrumentation. The authors describe 4 complex upper cervical spine cases in which the $\mathrm{O}$-arm combined with the StealthStation surgical navigation system was used to accurately place occipital screws, C-1 screws anteriorly and posteriorly, C-2 lateral mass screws, and pedicle screws in C-6. This combination was also used to navigate through complex bony anatomy altered by tumor growth and bony overgrowth. The 4 cases presented are: 1) a developmental deformity case in which the C-1 lateral mass was in the center of the cervical canal causing cord compression; 2) a case of odontoid compression of the spinal cord requiring an odontoidectomy in a patient with cerebral palsy; 3 ) a case of an en bloc resection of a C2-3 chordoma with instrumentation from the occiput to C-6 and placement of C-1 lateral mass screws anteriorly and posteriorly; and 4) a case of repeat surgery for a non-union at $\mathrm{C} 1-2$ with distortion of the anatomy and overgrowth of the bony structure at C-2. (http://thejns.org/doi/abs/10.3171/2014.1.FOCUS13514)
\end{abstract}

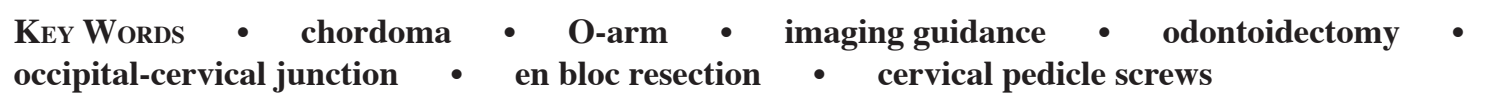

$\mathrm{P}$ LACEMENT of instrumentation from the occiput to the upper cervical spine has been challenging because of the complex anatomy and the need for a high degree of accuracy to avoid complications. The cervical region is prone to inflammatory, neoplastic, and traumatic conditions that can distort familiar anatomy, particularly due to the intimate relationship with the vertebral artery and spinal cord in limited bony structures. For the placement of instrumentation in the cervical spine, early use of direct visualization, serial radiography, and C-arm fluoroscopy have provided somewhat acceptable accuracy, but with the advent of intraoperative spinal navigation this accuracy has evolved. ${ }^{7}$ This has been enhanced with intraoperative $\mathrm{CT}$ combined with spinal navigation for the cervical spine ${ }^{10,17,19,24,25}$ and in particular the Oarm surgical imaging system (Medtronic). ${ }^{9,15,18}$ Nottmeier and Young ${ }^{15}$ described techniques for the placement of screws in the occiput, C-1 lateral masses, C-2 pars, and $\mathrm{C}-2$ laminae, using the $\mathrm{O}$-arm, and Ishikawa et al. ${ }^{9}$ described their experience with the $\mathrm{O}$-arm in the placement of cervical pedicle screws.

In this paper, we present 4 complex upper cervical spine cases in which the O-arm combined with the
Medtronic StealthStation surgical navigation system was used. Not only did this combination provide accurate placement of spinal instrumentation, but it also aided in bony decompression. The 4 cases involved 1) resection of a deformed C-1 lateral mass that was in the center of the cervical canal causing cord compression; 2) a transoral odontoidectomy; 3) an en bloc resection of a C2-3 chordoma; and 4) repeat surgery for a $\mathrm{C} 1-2$ nonunion in which the anatomy was distorted by bony overgrowth at $\mathrm{C}-2$.

\section{Case Reports}

Case 1

History and Examination. This 37-year-old woman presented to the clinic with tetraparesis, which had progressed to requiring wheelchair use. She also complained of left hand numbness and dropping objects from that hand. On neurological examination she had strength $5 / 5$ (manual muscle testing) for upper and lower extremities, with the exception of right wrist extension and hand grasp, which were $2 / 5$. She had upper- and lower-extremity hyperreflexia with bilateral Babinski and Hoffman 


\section{K. H. Guppy, I. Chakrabarti, and A. Banerjee}

signs. The patient had been seen at an outside hospital 2 years earlier and had been offered surgery but had decided against it.

Imaging. CT of the cervical spine (Fig. 1 left) showed that the right $\mathrm{C}-1$ lateral mass was rotated into the spinal canal. There was enlargement of the right occipital condylar region, possibly due to assimilation of the right lamina of the C-1 ring. Congenital fusion of the C5-7 and T1-3 vertebral bodies was also seen, with associated fusion of a number of the spinous processes. 3D reconstruction of a CT angiogram showed the right C-1 lateral mass rotated into the foramen magnum with the right vertebral artery entering the lateral mass, as well as the left hemilamina of C-1 (Fig. 2A).

Magnetic resonance imaging of the cervical spine (Fig. 1 right) showed dislocation of C-2 with respect to $\mathrm{C}-1$, with basilar invagination and horizontal orientation of the clivus. There was downward herniation of the cerebellar tonsils and kinking of the proximal cervical cord with compression. Increased signal intensity was seen within the cord at the C3-4 level, consistent with myelomalacia. Congenital fusion was again seen for multiple cervical and upper thoracic vertebrae. Overall, there was progression of the basal invagination in comparison with MR images that had been obtained 2 years earlier.

Operation. The objective of surgery was decompression of the foramen magnum with prevention of further basilar invagination. One approach considered was a transoral approach with removal of the rotated C-1 ring from the canal with posterior stabilization from the occiput to C-7. An alternative approach was removal of the $\mathrm{C}-1$ ring posteriorly using image guidance with the $\mathrm{O}$ arm imaging system and occipital to cervical fusion. The latter was chosen.

The patient was placed in a prone position with the neck slightly flexed to allow access to the $\mathrm{C} 1-2$ junction. Her head was placed in a Mayfield skull clamp with pins
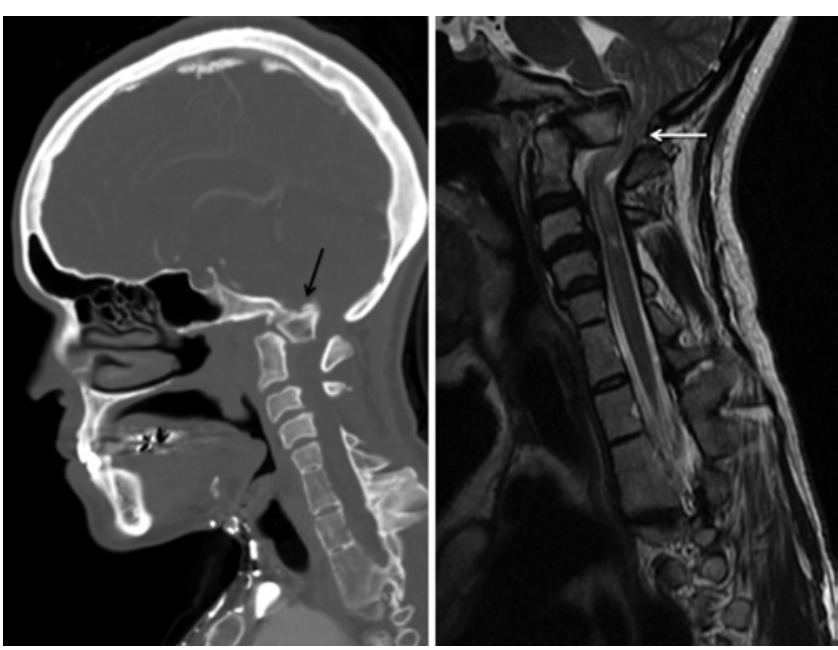

FIG. 1. Case 1. Preoperative imaging. Left: Sagittal CT reconstruction showing the right C-1 lateral mass in the foramen magnum (black arrow). Right: Sagittal T2-weighted MR image showing basilar invagination, kinking of the cord (white arrow), and the rotated right C-1 lateral mass. and the Mayfield Tri-Star swivel adaptor, which provided 2 additional starbursts to facilitate attachments for the reference arc for the StealthStation surgical navigation system. An incision was performed down to the fascia, and the suboccipital region and the laminae from C-1 to C-7 were exposed, with care being taken to expose the right side at C-1, which was missing half of the posterior arch.

The Medtronic StealthStation reference arc was attached to the Mayfield frame and a CT scan was performed from the base of the skull to T-1 using the Oarm. Intraoperative CT images were transferred to the Medtronic StealthStation.

Using the surgical navigation system, the depth of the screws for the keel in the occipital region was determined (Fig. 3A). Three screws were placed: one $16 \mathrm{~mm}$ in length and two $8 \mathrm{~mm}$ in length. All showed good purchase; tapping was performed in the usual fashion, and then the screws were placed. Using the CT image guidance, the entry points were determined for the C-7 pedicles (Fig. 3B). Using a StealthStation-guided Lenke probe, the C-7 pedicles were cannulated and tapped and $4.5 \mathrm{~mm} \times 22-$ $\mathrm{mm}$ screws were placed bilaterally. The guidance system allowed accurate placement of the screws without the need for a laminotomy. Lateral mass screws $(14 \mathrm{~mm}$ in length) were placed using the Magerl technique bilaterally at C-3, C-4, C-5, and C-6. The guidance system was again used in some instances to determine the lateral masses when landmarks were not visible as a result of bone overgrowth associated with the congenital fusions.

Attention was now drawn to the space between C-1 and $\mathrm{C}-2$ on the right side. The microscope was brought in, and, using blunt dissection, the right C-2 nerve root was identified. It was then ligated and cut. This allowed access to the underlying lateral mass. Using the CT guidance, the right lateral mass was identified (Fig. 3C). The right vertebral artery was identified and was found to enter the lateral mass laterally. Using the Sonopet ultrasonic aspirator (Stryker), a trough was created in the center of the right lateral mass, and then the thin lateral bony wall was pushed down into the trough and removed, with care being taken not to move the cord. The limit for the lateral border for the lateral mass bone removal was the foramen created for the right vertebral artery in the anomalous lateral mass. The CT guidance helped in identifying these borders. Bone was removed until the tip of the clivus was seen (Fig. 3D).

Another intraoperative CT scan was obtained, and this showed complete removal of the right lateral mass and good placement of all the screws. Rods were fashioned with hinges at C-1 and C-2 and were placed through the occiput, lateral mass, and pedicle screws. The occiput to C-7 was decorticated including the left lamina of C-1. Bone autograft was placed.

Outcome. Immediately after surgery, the patient began to regain strength in her upper and lower extremities. At the end of the 1st year she was walking with a cane. She continued to gain strength, and by the end of the 2nd year she was walking independently. Follow-up MRI showed decompression of the cord (Fig. 4). 
Intraoperative navigation for complex upper cervical surgery
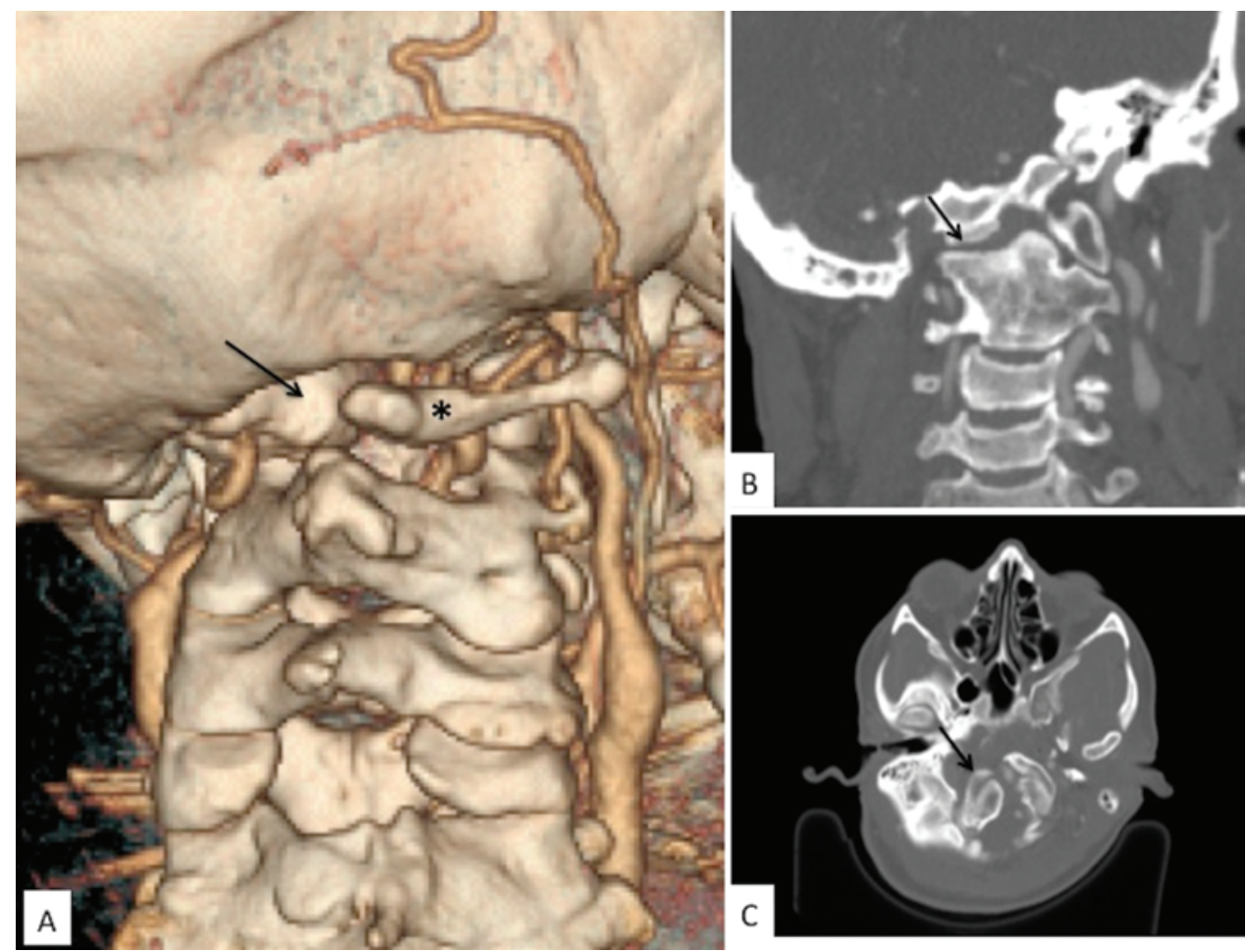

FIG. 2. Case 1. Preoperative $C T$ angiography. A: $3 \mathrm{D}$ reconstruction showing the right $\mathrm{C}-1$ lateral mass rotated into the foramen magnum (arrow) and the left hemilamina of $\mathrm{C}-1$ (indicated by asterisk). B: $2 \mathrm{D}$ reconstruction anterior-posterior view of cervical spine showing absence of right C-1 lateral mass (arrow). C: Axial view at the C-1 level with rotated right lateral mass in the canal (arrow).
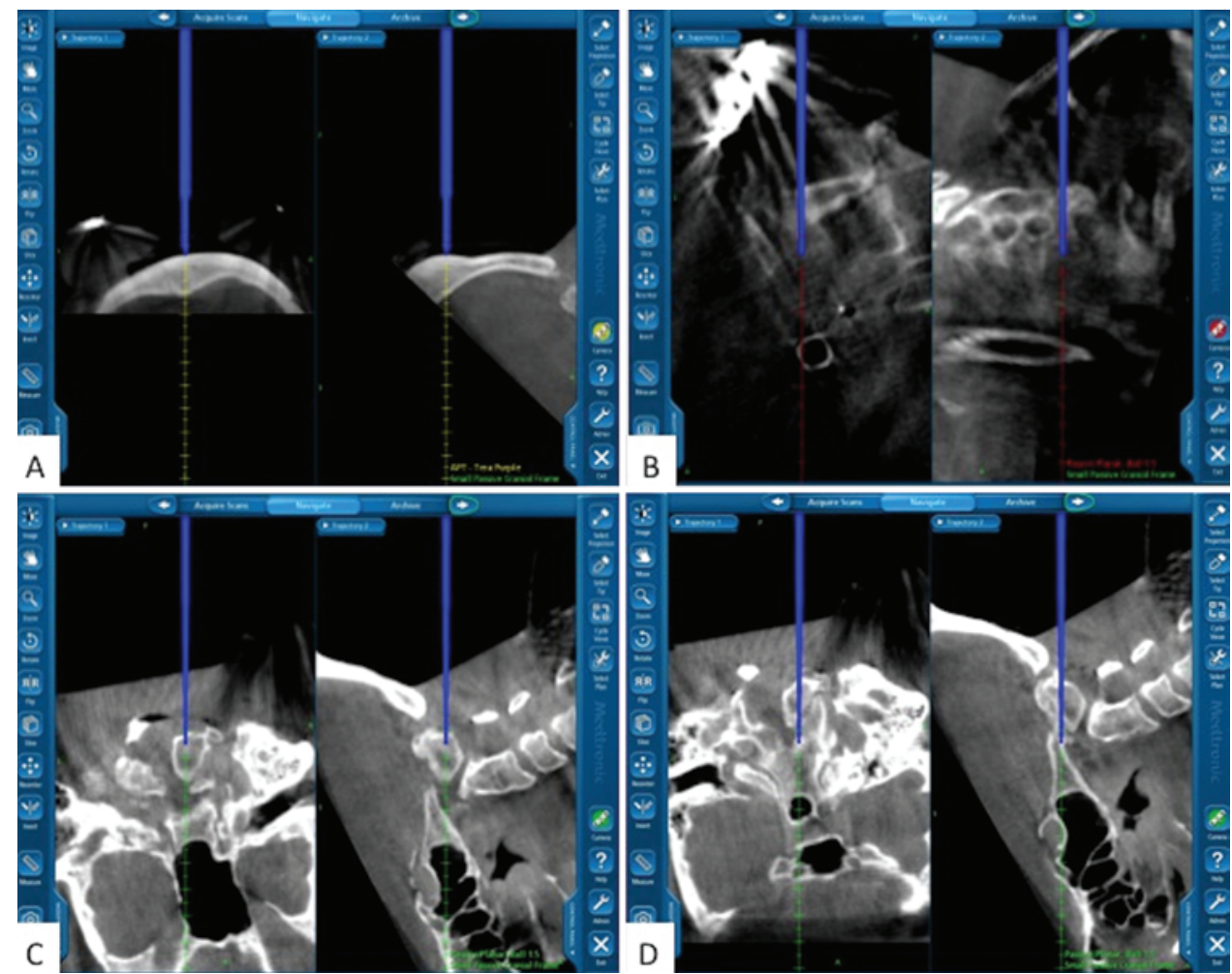

FiG. 3. Case 1. Intraoperative StealthStation screenshots showing O-arm-generated CT scans. A: Axial and sagittal views of keel of occiput used to estimate length of screws for placement of the occipital plate. B: Axial and sagittal views of C-7 for placement of bilateral pedicle screws. C: Axial and sagittal views obtained before removal of right lateral mass. D: Axial and sagittal views obtained after removal of right lateral mass. The pointer is at the tip of the clivus. 


\section{K. H. Guppy, I. Chakrabarti, and A. Banerjee}

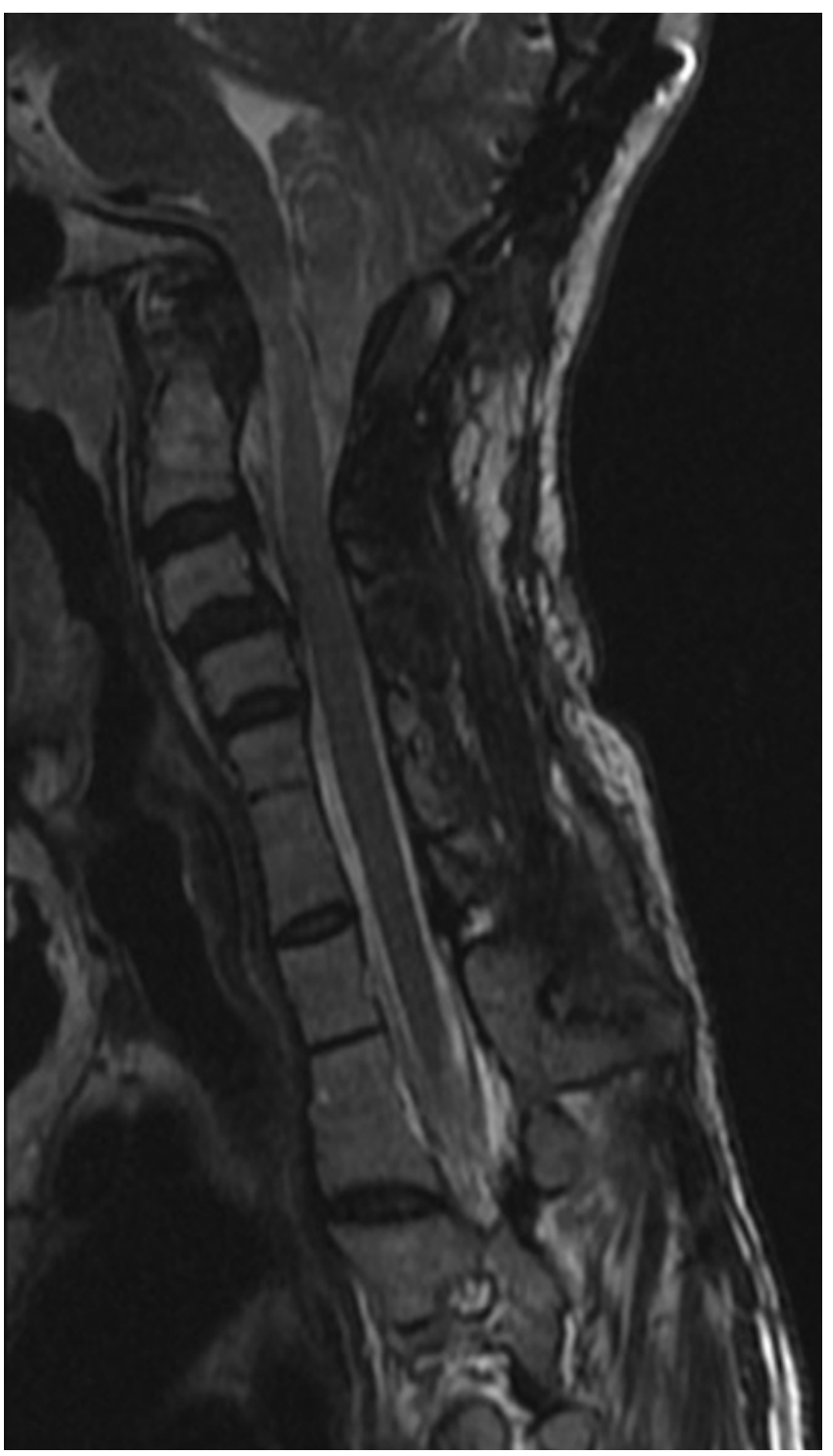

FIG. 4. Case 1. Postoperative sagittal T2-weighted MR image obtained at 2-year follow-up visit showing absence of spinal cord kinking and removal of rotated $\mathrm{C}-1$ lateral mass.

Case 2

History and Examination. This 49-year-old woman with Down syndrome had begun to have difficulty walking and using her hands 2 years previously. Until that time she had been able to live independently. More recently she had become wheelchair bound and had experienced increasing hand weakness and inability to sit up in the wheelchair. She denied neck pain. Manual muscle testing showed strength of 4/5 in her right biceps and triceps and $5 / 5$ for all other right upper- and lower-extremity testing. On her left side, both upper- and lower-extremity strength were 4/5. She had Babinski and Hoffman signs on the left; findings on the right side were equivocal. Proprioception was absent on the left side.

Imaging. CT of the cervical spine (Fig. 5 left) showed a chronic unhealed fracture or nonunion ossicle involving
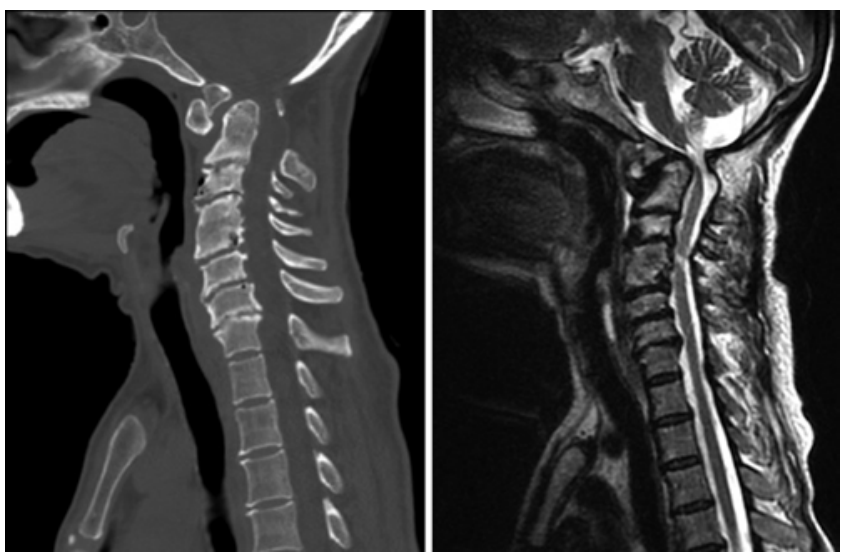

FIG. 5. Case 2. Preoperative images. Left: Sagittal CT reconstruction showing basilar invagination with the odontoid extending into the spinal canal. Right: Preoperative sagittal T2-weighted MR image of cervical spine showing kinking of spinal cord due to $\mathrm{C}-2$ angulation and basilar invagination.

the tip of the dens. Basilar invagination and widening of the predental interval suggested atlantoaxial instability. MRI of the cervical spine (Fig. 5 right) showed a narrow foramen magnum secondary to the posteriorly displaced odontoid. The tip of the odontoid process appeared to be separated, with marked posterior displacement of the remainder of the odontoid process. The anterior margin of the remainder of the distal portion of the odontoid was almost at the level of the posterior margin of the odontoid tip. There was marked attenuation of the upper cervical cord (Fig. 5 right) just below the medulla along the foramen magnum and kinking of the cord at the craniocervical junction.

Operation. It was decided that the best surgical approach was a 2-stage procedure, with a transoral approach for resection of the odontoid followed by an occipital-tocervical fusion. Intraoperative CT using the O-arm with image guidance was used in both procedures.

A tracheostomy was performed and the patient's head was then placed in a Mayfield frame with the neck slightly extended. The StealthStation reference arc was attached to the Mayfield frame. The patient was prepared and draped in the usual fashion with towels around the mouth and nares. Using the O-arm, an intraoperative CT scan of the cervical spine was obtained for navigation. This was done prior to placement of the Dingman retractor to avoid artifact that would prevent visualization of the spine. Once the retractor was placed with the tongue inferiorly placed and the cheeks retracted out laterally, the pharyngeal musculature was exposed. A suture was placed in the uvula to a red rubber catheter, which was pulled through the nostril to elevate the uvula superiorly.

An intraoperative CT scan was performed, and the anterior arch of C-1 was identified using the StealthStation guidance system (Fig. 6A). An incision was made with a Bovie cautery in the midline of the pharynx, followed by lateral spreading of the mucosal and then the muscular layers of the pharyngeal wall. A self-retaining retractor was placed. Using the CT guidance, the C2-3 disc space (Fig. 6B) was found in order to identify the 


\section{Intraoperative navigation for complex upper cervical surgery}
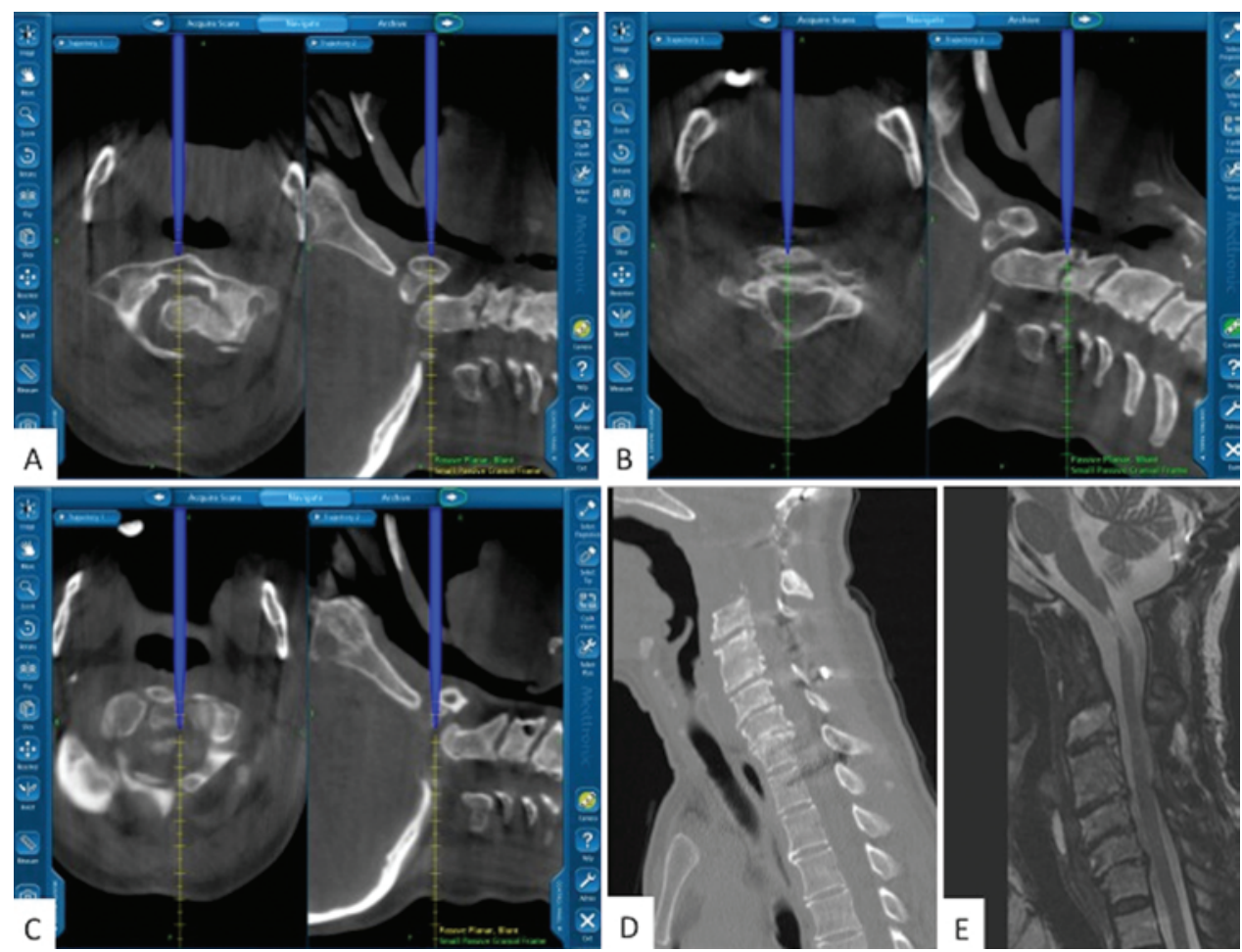

FIG. 6. Case 2. Intraoperative and postoperative images. A-C: StealthStation screenshots of intraoperative O-arm-generated CT images in axial and sagittal views showing the planned approach at the top of the odontoid $(A)$, the extent of planned resection to the bottom of the odontoid $(B)$, and the depth of the odontoid resection (C). D: Postoperative sagittal CT reconstructed view showing resected odontoid. E: Postoperative (2 years) sagittal T2-weighted MR image of cervical spine showing absence of kinking of spinal cord.

extent of the resection. The C-1 arch was clearly protruding anteriorly under the microscope. This was drilled and removed in a piecemeal fashion, exposing the os odontoideum below it. The os odontoideum was also removed in a piecemeal fashion revealing thickened, hypertrophied ligaments. The ligaments were removed, and the dens was exposed and cored out from the center until the posterior wall was identified (Fig. 6C). Navigation was used during the entire decompression process to aid in defining the extent of resection of the odontoid superiorly, inferiorly, and laterally. The posterior part of the C-2 body was very calcified and was fused to the posterior longitudinal ligament, which was fused to the dura. Using the diamond drill, bone was removed until only a thin calcified layer was left on the dura in a few areas. Another intraoperative CT scan was obtained on completion of removal of the odontoid; it showed complete bony removal of anterior arch of C-1, os odontoideum, and odontoid. The wound was closed in the usual fashion in multiple layers. The patient was placed in a halo orthosis until the posterior fusion procedure.

The second-stage procedure was performed 2 days later. The patient was placed in a prone position in the Mayfield frame, which was attached to the operating table. An incision was made in the midline of the posterior cervical region. Subperiosteal muscle dissection was completed, exposing the suboccipital region down to the T-2 level. An intraoperative CT scan was performed using the $\mathrm{O}$-arm. Using $\mathrm{CT}$ guidance, occipital screws were placed in the occipital keel for placement of the DePuy occipital plate. Using the Harms and Melcher technique with CT guidance aiming toward the anterior arch of C-1, screws were placed. Similarly, using CT guidance, pedicle screws were placed at T-1 and T-2 bilaterally. Lateral mass screws were placed at C-3, C-4, C-5, and C-6 bilaterally, using the Magerl technique without CT guidance. A laminectomy was completed at C-1. Similarly, laminectomy was performed at C-4 and C-5 because preoperatively there was cord myelomalacia at the C-4 level (Fig. 5 right). Tapered rods were placed bilaterally from the occiput to $\mathrm{T}-2$.

Bony fusion was accomplished by using iliac crest graft, morsellized lamina, and allograft. Iliac crest graft was placed between C-2 and the occiput. The wound was closed in the usual fashion. A final intraoperative CT scan was obtained to verify good placement of the screws.

Outcome. Soon after surgery, the patient was able to sit up in a wheelchair, with increasing use of her hands. With time she advanced to standing and walking with minimal assistance during the 1st year after surgery. By the 2nd year she had moved into her own home and is now living independently. Two-year follow-up imaging showed complete removal of the odontoid (Fig. 6D) and removal of kinking of the cord with residual myelomalacia (Fig. 6E).

Case 3

History and Examination. This 49-year-old woman 


\section{K. H. Guppy, I. Chakrabarti, and A. Banerjee}

initially presented with difficulty breathing in a recumbent position, especially at night. She also complained of neck pain and radicular pain in her right shoulder. A plain cervical spine radiograph showed soft-tissue abnormality, so an MRI study was ordered and the patient was referred for neurosurgical evaluation. The results of neurological examination were normal.

Imaging. MRI of the cervical spine (Fig. 7) showed a large homogeneously enhancing mass centered at the C-2 level and displacing the cervical cord to the left, causing severe narrowing of the canal. At the level of the posterior pharynx, the tumor extended toward the left of midline and involved the right longus coli muscle. A CT angiogram showed the right vertebral artery at the C2-4 levels with poor filling and encased by the mass at the C-3 level. The right cervical internal carotid artery was not encased by the mass but was displaced.

Surgery. The preoperative workup included a biopsy of the C-2 mass, which showed histological features and immunohistochemical characteristics consistent with chordoma. An en bloc resection of the tumor was planned; this required sacrificing the right vertebral artery at $\mathrm{C}-1$ to C4-5 with GDC fiber coils as well as designing a unique anterior cage to accommodate the lateral masses of C-1 through C-4.

In summary, surgery required 2 stages. The first stage consisted of a posterior cervical approach with 1) C2-3 laminectomy and facetectomy; 2) cutting of the right $\mathrm{C}-2, \mathrm{C}-3$, and $\mathrm{C}-4$ nerve roots and ligation of the right vertebral artery (which was embolized before the procedure) at the $\mathrm{C} 1-2$ and $\mathrm{C} 3-4$ levels; 3) unroofing of the left vertebral artery from C-2 to C-3; 4) placement of the DePuy occipital plate, lateral mass screws at C-1, C-4, and $\mathrm{C}-5$ and bilateral pedicle screws at C-6; and 5) bone fusion with allograft and rib autograft. Details are well described in Guppy et al. ${ }^{6}$

Intraoperative CT using the O-arm imaging system and the StealthStation surgical navigation system was es-
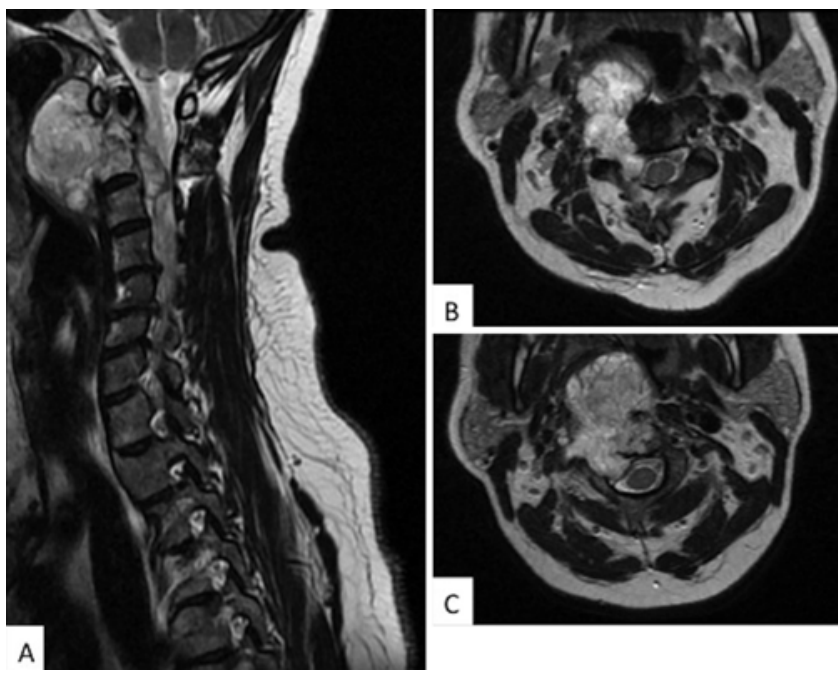

FIG. 7. Case 3. Preoperative images. Sagittal (A) and axial (B and C) T2-weighted MR images showing a chordoma at the C-2 and C-3 levels. sential in determining the bony anatomy for resection of the tumor as well as placement of hardware. The patient was placed in a Mayfield head frame attached to the operating table in the prone position. Dissection was completed from the occiput to C-7. Using the intraoperative $\mathrm{CT}$, the thickness of the skull in the midline in the suboccipital area was determined and the holes were drilled and tapped and the screws placed (Fig. 8A). Identifying the lateral masses at $\mathrm{C}-1$ and locating the right vertebral artery was easily done using the guidance system (Fig. $8 \mathrm{~B})$. Once normal anatomy was not visible after removal of some bone from the lateral masses on the left side, the pointer for the guidance system (Fig. 8C) was useful for identifying the inferior lateral masses and transverse foramina. This allowed safe bone removal (Fig. 8D) using the ultrasonic bone curette (Sonopet ultrasonic aspirator). Lateral mass screws were placed, using the Magerl technique, bilaterally at C-4 and C-5. At C-6 we used the $\mathrm{O}$-arm CT images with a StealthStation guided Lenke probe to cannulate the C-6 pedicles bilaterally (Fig. 9C) and placed pedicle screws. At C-1, the CT guidance was used to place a conventional lag screw posteriorly in the left lateral mass (Fig. 9C). We attempted to place a screw on the right lateral mass, but it appeared that the screw had to be placed more laterally to accommodate the anterior $\mathrm{C}-1$ screw and we found the purchase to be less than desirable. Because we felt it was more important to have the anterior screws in place, we abandoned the placement of this screw.

The second stage of surgery was undertaken 4 days later and consisted of 1) a tracheostomy; 2) a right transcervical, transmandibular, and transpalatal approach; 2) en bloc resection of the $\mathrm{C} 2-3$ tumor; 3 ) placement of the predesigned cage with a plate extending from $\mathrm{C}-1$ to $\mathrm{C}-4$ with autograft; and 4) iliac crest harvest.

After a standard tracheostomy was completed, a right transcervical, transmandibular, and transpalatal approach was performed. The tumor was isolated and the C3-4 disc was removed under the surgical microscope. The posterior longitudinal ligament was cut and the uncovertebral joints were removed. Using the CT guidance, the anterior tubercle of the transverse process at C-3 was identified, and using the Sonopet ultrasonic aspirator, the vertebral artery was skeletonized and removed from the foramen transversarium, which had been opened previously in Stage 1 posteriorly. The CT guidance system was also used to identify the left foramen transversarium at C-2 and the ultrasonic aspirator was used to remove the portion of the transverse process anterior to the foramen. The vertebral artery was mobilized and slight traction was placed on it to move the artery laterally away from the $\mathrm{C}-2$ and $\mathrm{C}-3$ vertebral bodies.

On the right side, the tumor was peeled off the anterior arch, maintaining its capsule, and reflected to the right side, exposing the $\mathrm{C} 1-2$ facets on the right and left sides. Using a Penfield No. 4 dissector, the articular capsule was opened and the facets were disarticulated. The anterior arch of C-1 was then cut using the matchstick drill, exposing the odontoid process. The odontoid was then circumferentially decompressed by using a scalpel and No. 1 Kerrison punch to cut the alar ligament, apical 


\section{Intraoperative navigation for complex upper cervical surgery}
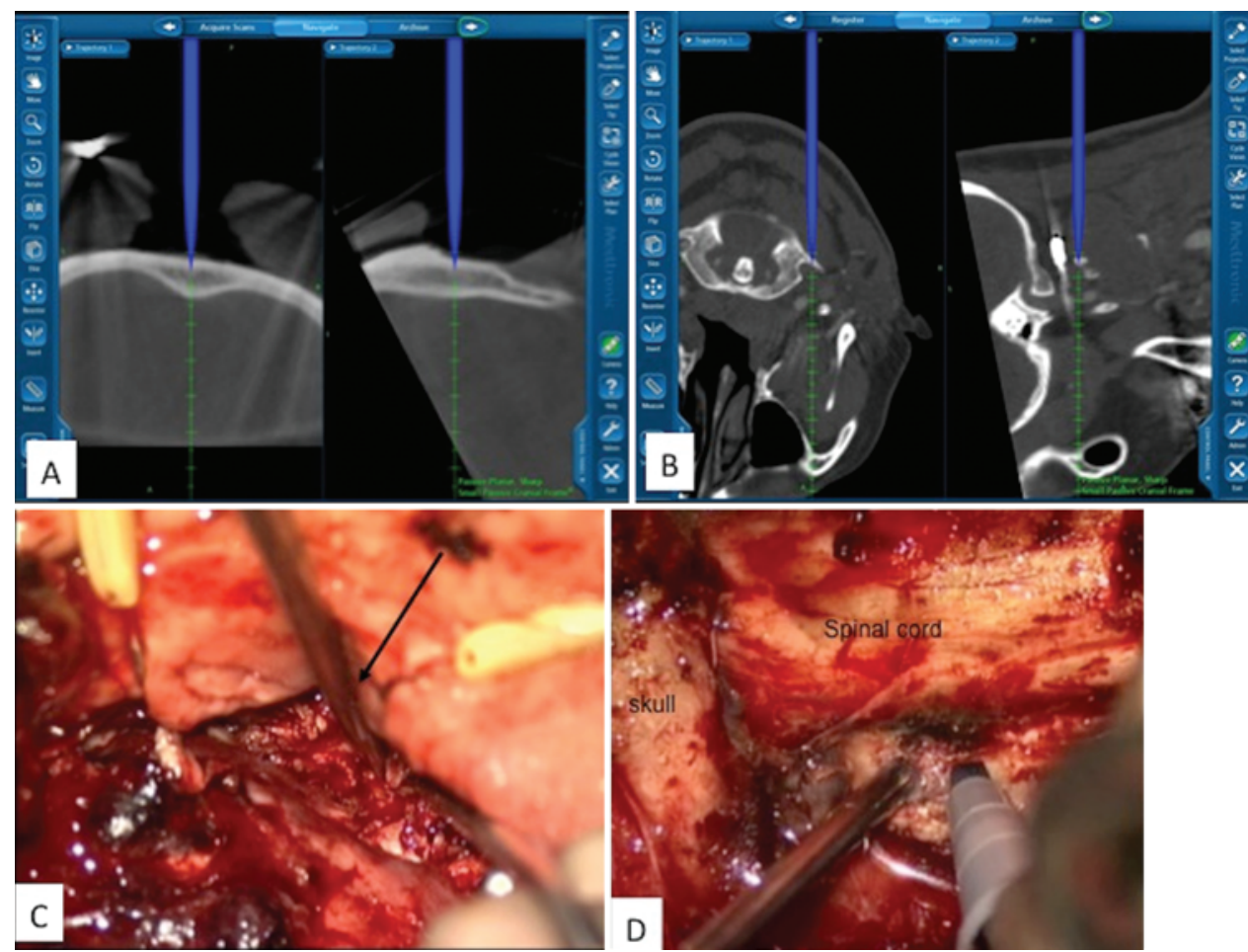

FIG. 8. Case 3. Intraoperative images. A and B: StealthStation screenshots of O-arm-generated CT scans in axial and sagittal views showing the thickness of the keel of the skull $(A)$ and the use of guidance to outline the left posterior tubercle of $C$ - 1 (B). C and D: Intraoperative views showing pointer (indicated by arrow) being used to outline the left lateral masses (C) and use of the Sonopet ultrasonic bone aspirator to resect the left lateral masses (D).

ligament of the dens, tectorial membrane, posterior longitudinal ligament, and transverse ligament of the atlas. By lifting $\mathrm{C}-2$ and $\mathrm{C}-3$ while disarticulating the $\mathrm{C} 1-2$ facets, the entire tumor was removed with the body of $\mathrm{C}-2$, including the odontoid, and C-3, exposing the underlying spinal cord.

Iliac crest graft was harvested and placed in the prefabricated titanium cage. The superior endplate of C-4 and inferior lateral masses of C-2 were decorticated, and the cage was placed between $\mathrm{C}-1$ and $\mathrm{C}-4$ (Fig. 9A and B). Using the CT guidance an anterior C-1 screw (4 mm $\times 14 \mathrm{~mm}$ ) was placed on the left side and a larger $4 \mathrm{~mm} \times$ $20 \mathrm{~mm}$-screw was placed on the right side (Fig. 9C). Two $4 \mathrm{~mm} \times 14 \mathrm{~mm}-$ screws were placed in C-4 to secure the distal end of the cage. Once the hardware was secured, the pharyngeal wall was closed and the mandible reattached. The incision was closed in multiple layers.

Outcome. The patient was out of bed and ambulating by postoperative Day 2. She had some transient right arm weakness that resolved in a few days. She was placed in a halo vest for 3 months and by 6 months had no neck pain and was functioning independently, as she did prior to surgery. A follow-up CT scan performed 2 years after en bloc resection of the chordoma showed bone growth in the cage with the construct intact (Fig. 9).

\section{Case 4}

History and Examination. This 44-year-old man presented with complaints of constant dull, stabbing pain in the neck and numbness in the fingers and feet, which had developed after he was involved in a recent motor vehicle accident. He had undergone a $\mathrm{C} 1-2$ fusion procedure 25 years previously and had experienced neck pain for a greater part of the time since, but it had worsened after the accident. More recently on flexing his neck he had felt shooting pain down both arms. On neurological examination he showed normal strength with no evidence of Hoffman sign, reproducible L'Hermitte sign, or Babinski sign. His deep tendon reflexes were normal.

Imaging. Flexion-extension radiographs showed 2-3 $\mathrm{mm}$ of movement at $\mathrm{C} 1-2$. MRI showed artifact at C1-2, with poor visualization of the vertebrae. CT myelography of the cervical spine showed anterior displacement of the $\mathrm{C}-1$ arch with some narrowing at the cervicooccipital junction (Fig. 10A). An axial view of C-2 (Fig. 10B) showed overgrowth of bone with wires in the spinous process. There was no evidence of a bony fusion between C-1 and C-2, and broken wires could be seen.

Operation. The patient had a symptomatic nonunion at $\mathrm{C} 1-2$ and it was decided to redo the fusion with $\mathrm{C}-1$ lateral mass screws. Because of prominent vertebral arteries, translaminar screws were placed at C-2. Intraoperative $\mathrm{O}$-arm $\mathrm{CT}$ was used for navigation guidance in placement of the $\mathrm{C}-1$ and $\mathrm{C}-2$ screws.

After the patient was intubated, a Mayfield frame was applied to his head, and he was placed prone with his head in neutral position. His previous incision was opened to the fascia, and then the C-2 and C-3 spinous process as well as the suboccipital region were exposed. 

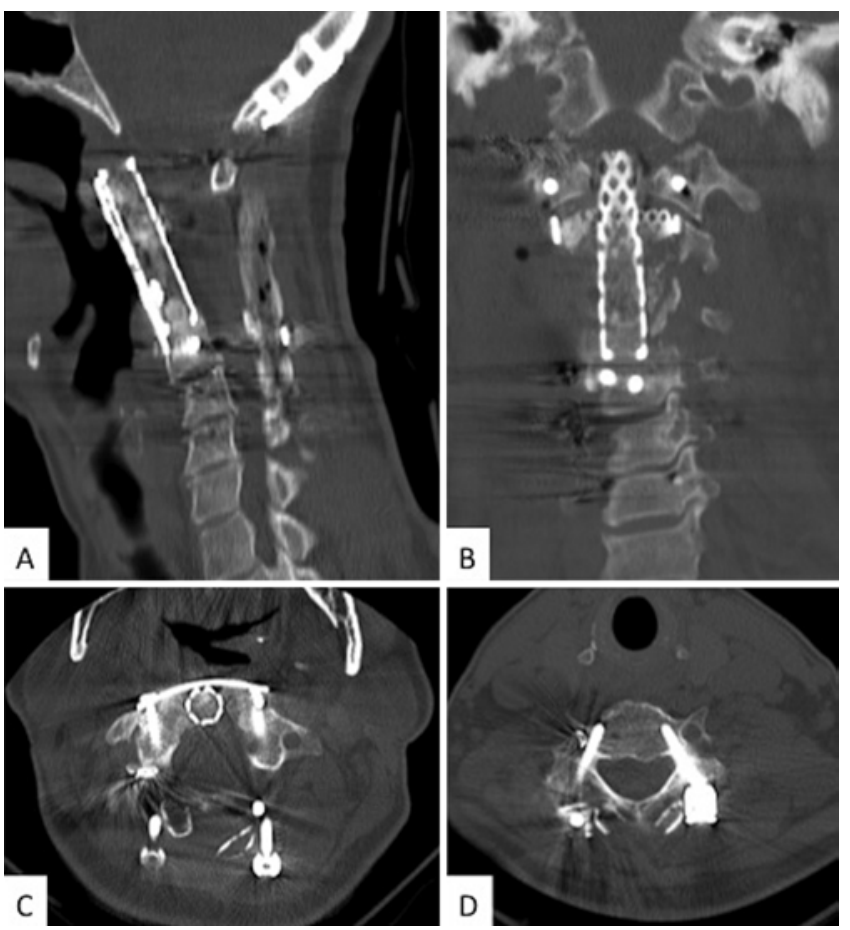

FIG. 9. Case 3. Postoperative CT images obtained 2 years after surgery. A: Sagittal reconstruction showing the anterior cage with bone growth in the cage. B: Coronal reconstruction showing the cage with lateral masses sitting below the C-1 lateral masses. C: Axial view of C-1 with anterior screws and a single (left) posterior screw. D: Axial view of C-6 with bilateral pedicle screws.

There was a large mass incorporating $\mathrm{C}-2$ and overlying C-1 but not attached to it. It was difficult to determine the normal landmarks, so the StealthStation reference arc was attached to the Mayfield frame and an intraoperative CT scan was performed with the O-arm. Using the guidance system we were better able to identify details of the bony complex. The C1-2 nonunion was found, with broken wires between the vertebrae. The wires were removed without any difficulty and with no evidence of cerebrospinal fluid leak.

The posterior arch of C-1 was identified using CT guidance. Some of the superficial bone/fibrous tissue was removed by blunt dissection and the use of Kerrison punches. The inferior facets of the joints at $\mathrm{C}-1$ and $\mathrm{C}-2$ were identified bilaterally. Using CT guidance as described previously, the C-1 screws $(20 \mathrm{~mm}$ in diameter with a 1-cm shank) were placed bilaterally.

Because of the overgrowth of bone on the C-2 lamina, the normal anatomical landmarks were difficult to identify. Using CT guidance, translaminar screws were placed at C-2. The entry points were selected so as to allow the screws to be placed without interfering with each other and to obtain a trajectory that maximized the thickness of the lamina and the new bony formation (Fig. 10C). Thirty-millimeter screws were used bilaterally.

The posterior arch of C-1 was identified and, using the drill as well as Kerrison punches, the lamina was removed. Simultaneously, iliac crest graft was obtained and placed between the $\mathrm{C}-2$ spinous process and $\mathrm{C}-1$ and on top of the remaining $\mathrm{C}-1$ portion of the posterior arch. It

\section{K. H. Guppy, I. Chakrabarti, and A. Banerjee}

was held in place using Dacron-0 suture. The C1-2 facets were decorticated and iliac bone was placed between them.

A final intraoperative CT was obtained using the $\mathrm{O}$ arm, confirming that the screws were well placed in C-1 and $\mathrm{C}-2$. The graft attached to $\mathrm{C}-1$ did not show compression at that level. The wound was closed in the usual fashion.

Outcome. The patient had relief of his symptoms within 2 weeks of surgery. His neck pain resolved, and by the 3 rd postoperative month he was able to return to work. A CT scan obtained at the 2-year follow-up visit showed the laminar screws in place (Fig. 10D).

\section{Discussion}

There is no doubt that intraoperative spinal imaging and navigation has been advanced with the introduction of the O-arm surgical imaging system and StealthStation navigation system..$^{11}$ The technology encompasses fluoroscopically acquired $2 \mathrm{D}$ images with a full $360^{\circ}$ scan that is used for 3D volumetric reconstruction. An integrated LED (light-emitting diode) tracking system that incorporates the $\mathrm{O}$-arm with the reference arc placed on the patient allows for automated registration as the images are acquired and provides the navigation component for the surgeon. ${ }^{7}$

The use of the O-arm with StealthStation guidance has been shown to be invaluable in increasing the accuracy of pedicle screw placement in the lumbar and sacral spine when compared with freehand placement, ${ }^{21}$ as well as in pedicle screw placement in the thoracic and lumbosacral spine when compared with fluoroscopy-guided placement. ${ }^{20,22}$ More recently it was used in the placement of distal pelvic fixation with bilateral S-2 alar iliac fixation. ${ }^{16}$ It has also been shown to reduce operative time, in comparison with the use of preoperative CT scans with image guidance, ${ }^{2}$ and to reduce radiation exposure compared with fluoroscopic guidance..$^{14}$ Performing intraoperative $\mathrm{CT}$ at the end of the surgical procedure also reduces the need to return patients to the operating room. ${ }^{23}$ The $\mathrm{O}$-arm has also been used in minimally invasive spine surgery ${ }^{1,3}$ including endoscopic surgery for resection of chordomas ${ }^{8}$ and intraoperative CT-navigated lateral interbody fusion procedures. ${ }^{4}$ Its role in the cervical spine, however, has been limited, both for anterior cervical surgery and posterior cervical fusion. ${ }^{9}, 15,18$ The 4 cases presented in this paper illustrate new applications in the use of the $\mathrm{O}$-arm for image guidance in the upper cervical spine.

\section{Posterior Upper Cervical Spine Surgery Using Image Guidance}

Because of the intimate spatial relationship between the vertebral artery and spinal cord in the upper cervical region, precise placement of screws is essential. Nottmeier and Young ${ }^{15}$ described a technique for placing screws at the occiput, $\mathrm{C}-1$, and $\mathrm{C}-2$ using 2 paired image guidance systems: 1) the BrainLAB VectorVision system in conjunction with the Arcadis Orbic Isocentric C-arm 


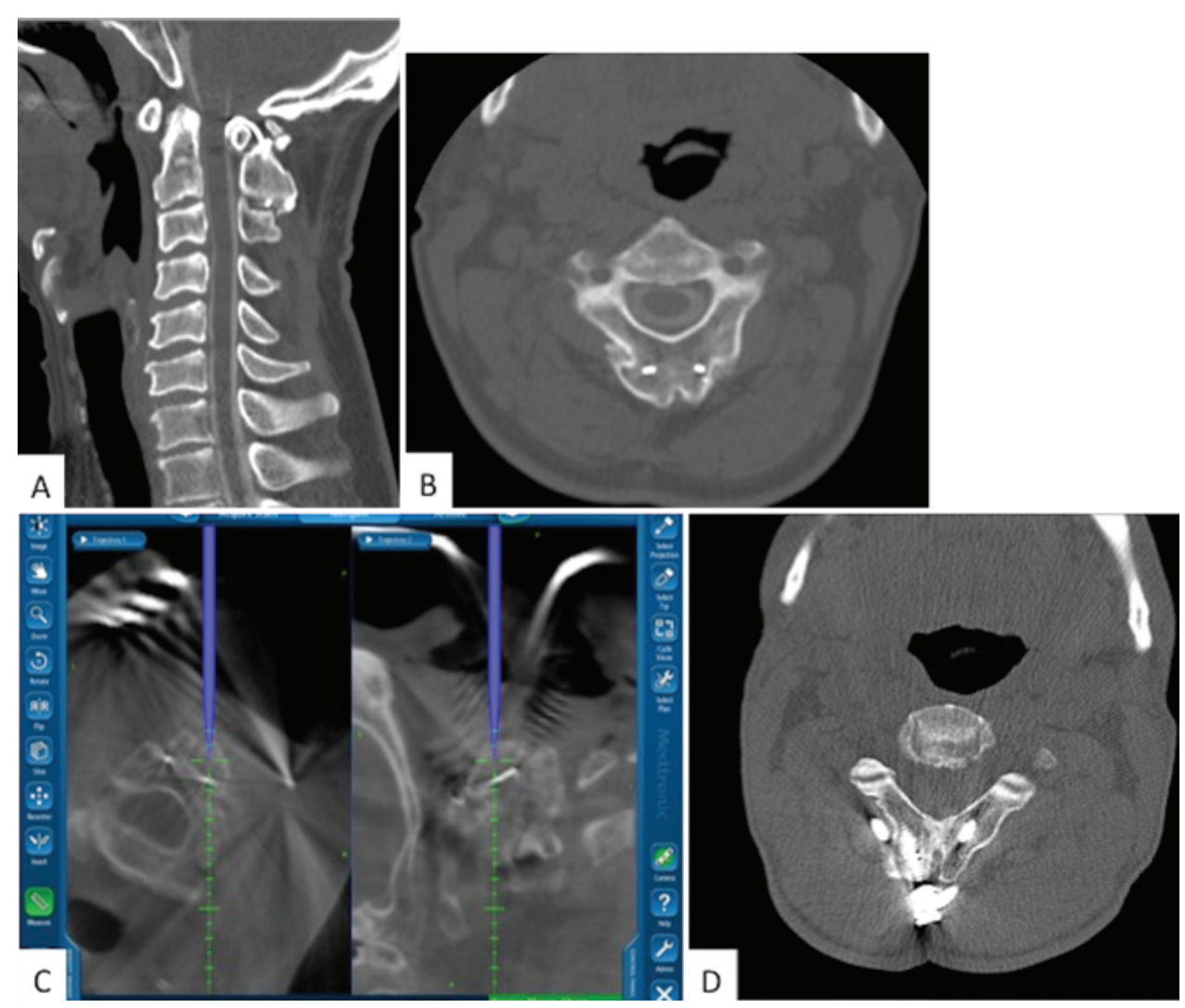

FIG. 10. Case 4. Preoperative, intraoperative, and postoperative images. A: Sagittal view CT myelogram showing C1-2 wires in place without $\mathrm{C} 1-2$ fusion and C-1 posteriorly displaced in canal. B: Axial CT image at the C-2 level showing bone formation around the wires and loss of normal anatomy. C: StealthStation screenshot showing axial and sagittal views of intraoperative 0-arm-generated CT scans for placement of translaminar screws at C-2. D: Axial CT image obtained 2 years postoperatively showing the bilateral translaminar screws.

(Siemens Medical Solutions), and 2) the O-arm paired with the Stealth Treon system (Medtronic). They reported on placement of a total of 82 screws, including 24 in the occiput, 24 in lateral masses of $\mathrm{C}-1,13$ in the $\mathrm{C}-2$ pars, and 21 in C-2 laminae, with only 1 screw with a minimal breach of the outer lamina of C-2.

Two years later, Yu et al. ${ }^{25}$ described 108 screw insertions performed for the treatment of complex craniovertebral junction malformations using a CT scanner installed in a preexisting operating room connected to an integrated neuronavigation system. There were $20 \mathrm{C}-1$ lateral mass screws, 26 C-2 screws, 14 C-3 or C-4 pedicle screws, and 32 occipital screws placed. Their accuracy, using image guidance, was $98.1 \%$.

Ishikawa et al. ${ }^{9}$ retrospectively reviewed 21 consecutive cases in which 108 cervical pedicle screws were placed using the $\mathrm{O}$-arm-based navigation system. They reported a minor pedicle violation rate of $8.3 \%$ (9 of 108 screws) and a major pedicle violation rate of $2.8 \%$ (3 of 108 screws). While these error rates may be low, we believe that if the misplacements had been found intraoperatively they could have been corrected. For this reason we have advocated performing another intraoperative CT scan before wound closure. Van de Kelft et al. ${ }^{23}$ repositioned approximately $1.8 \%$ of their screws that had been placed with CT-guided navigation.

In our series we used the O-arm to place 6 occiput screws (Cases 1 and 2), 5 posterior C-1 lateral mass screws (Cases 2, 3, and 4), 2 C-2 translaminar screws (Case 4), 2 C-6 pedicle screws (Case 3), 2 C-7 pedicle screws (Case 1), and 2 anterior lateral mass screws at C-1 (Case 3). Repeat intraoperative $\mathrm{CT}$ before wound closure showed no pedicle violations or malposition of any of the 19 screws we placed using the $\mathrm{O}$-arm system.

\section{Odontoidectomy Using Image Guidance}

Veres et al. ${ }^{24}$ in 2001 described their early use of navigation-assisted transoral odontoidectomy in 3 patients. Preoperatively the patients were placed in a halo device, with fiducial markers attached supraorbitally and to both mastoids, and a CT scan was obtained. Resection of the odontoid was accomplished using the BrainLAB VectorVision navigation system with little need for fluoroscopy. More recently, in 2012, Li et al..$^{10}$ described the use of a ceiling-mounted frameless infrared-based neuronavigation system (VectorVision) and 40-multislice CT scanner mounted in an operating room in 19 cases involving patients with complex craniovertebral junction malformations. Eighteen of these patients had a transoral approach with image guidance combined with endoscopy.

There have been no reported cases in which the Oarm for was used for odontoidectomies. In this article, we present, for the first time, the use of CT image guidance 


\section{K. H. Guppy, I. Chakrabarti, and A. Banerjee}

(O-arm), as shown in Case 2, by defining the superior and inferior margins as well as the depth for complete removal of the odontoid. We have also found that a second intraoperative CT scan is necessary to evaluate the bony decompression. This is far superior to the technique described by Mummaneni and Haid ${ }^{12}$ in which iohexol dye was placed into the resection cavity after an odontoidectomy and a lateral fluoroscopic image was obtained to confirm the extent of decompression. The authors found that the spread of the dye helped to reveal any remaining remnant of the dens. Li et al. ${ }^{10}$ also described the usefulness of a second intraoperative CT study in their Patient 5 in whom a postdecompression CT was performed and a small residual bone spicule compressing the neuraxis was found. The spicule was removed, and a third intraoperative 3D-CT showed that sufficient anterior decompression had been achieved.

\section{Chordoma Resection Using Image Guidance}

Chordoma resections in the cervical spine can be difficult due to the predilections of these tumors to occur near delicate and vital structures and their destructive behavior, which can obscure familiar bony landmarks. Hsu et al. ${ }^{8}$ described the use of an image-guided, endoscopic, transcervical approach for resection of a recurrent chordoma at C1-2. They used the BrainLAB frameless stereotactic system to remove bone, tumor, and methylmethacrylate from a previous reconstruction. They did not place any spinal instrumentation using image guidance. En bloc resection of chordomas with negative margins can be more difficult to perform without damaging adjacent structures and causing significant clinical morbidity. We previously described the technique of en bloc resection of an upper cervical chordoma using image guidance. ${ }^{6}$ The tumor distorted the normal anatomy, and CT guidance allowed en bloc resection without violating vital structures. In Case 3, we further describe details of the use of the $\mathrm{O}$-arm-based system for placement of the occipital screws, lateral mass screws at $\mathrm{C}-1$, and pedicle, as well as resection of the chordoma using image guidance.

\section{Revision Cervical Spine Surgery Using Image Guidance}

In situations where anatomical landmarks are difficult to identify, CT image guidance has been shown to be ideally suited. In patients with scoliosis, degenerative spine disease, or ankylosing spondylitis and in very obese patients or in treating areas of the cervicothoracic junction and upper thoracic spine where fluoroscopy has poor resolution, CT guidance is very useful with reduction in radiation exposure. ${ }^{5,18}$ This is especially true in revision cervical spine surgery where the normal anatomical landmarks are obscured. Seichi et al. ${ }^{19}$ used a frameless stereotactic image-guidance system with simultaneous fluoroscopy in 10 patients who required revision of previous cervical spine surgery. They found image guidance helped them to achieve rigid fixation and ensure safety. We also found it useful as illustrated in Case 4. The revision of the $\mathrm{C} 1-2$ nonunion in that case was challenging due to bony overgrowth that obscured the normal landmarks for the placement of the $\mathrm{C}-2$ laminar screws. In
Case 1 also, bony landmarks were not apparent, but this time it was due to a congenital deformity. In that case, the deformity in the lateral mass distorted the normal anatomy, and intraoperative CT was essential in locating the lateral mass and removing it.

\section{Technical Nuances}

Since we acquired the $\mathrm{O}$-arm surgical imaging system in 2006, several technical nuances have helped us to reduce the time to obtain imaging, to maintain a sterile environment, and to increase accuracy.

To reduce the time to obtain and transmit images, we have found it important to train our staff, as well as the surgeons, to become familiar with the equipment and to be able to troubleshoot error messages. As the radiology technicians became better versed with the use of the Oarm system, performing a CT scan and transferring images to the Medtronic StealthStation navigation system could be accomplished in 20 minutes.

Maintaining sterile conditions was also very important. Using a plastic cover for the O-arm "donut" (Fig. $11 \mathrm{~B}$ and $\mathrm{C}$ ) provided such an environment. We have found that covering the operative field with sterile drape sheets and then bringing the O-arm "donut" uncovered was faster and still maintained a sterile field.

Accuracy was achieved by proper placement of the reference arc as well as minimizing movement of the vertebrae. The placement of the reference arc increased our accuracy in screw placement if it was placed close to the structure of interest (Fig. 11). As Nottmeier and Pirris ${ }^{13}$ pointed out in their article, placement of the reference arc attached to the Mayfield frame provided accuracy in screw placement for the occiput to C-3 and C-4. The further away from the reference arc, the less accurate the placement was. A second intraoperative CT scan may need to be obtained with the arc placed on a cervical spinous process. In our series only one intraoperative CT

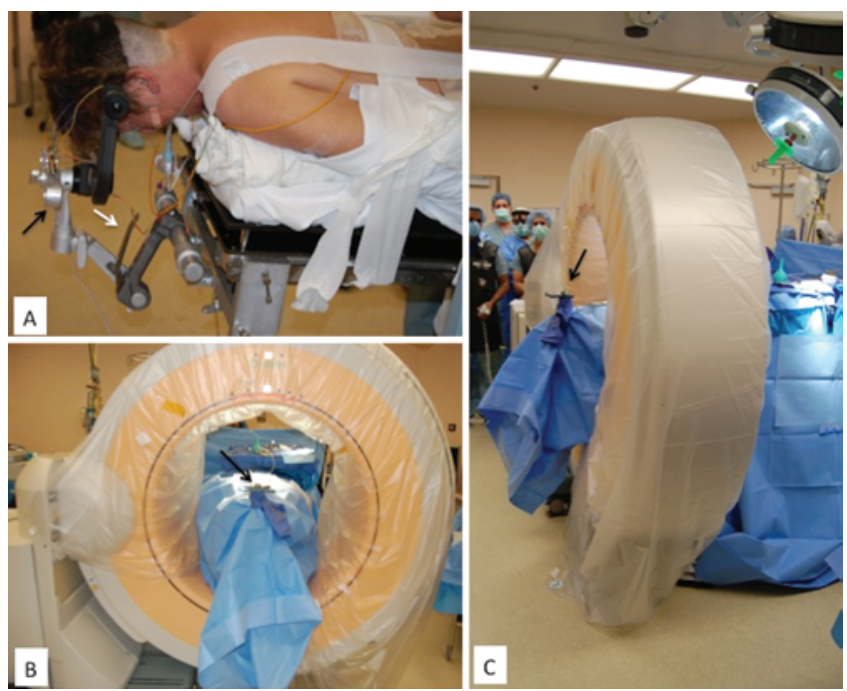

FIG. 11. O-arm and patient positioning. A: Patient in Mayfield head clamp with support/mounting device (white arrow) reversed and with Mayfield Tri-Star swivel adaptor (black arrow). B and C: Patient in low profile to fit into the "donut" of the 0-arm, with reference arc (black arrow) attached to Tri-Star swivel adaptor. 


\section{Intraoperative navigation for complex upper cervical surgery}

scan was needed for placement of screws in all cases but Case 2; in Case 2, pedicle screws were placed in the upper thoracic spine, requiring the arc to be placed on $\mathrm{C}-7$ for a second intraoperative CT. Also, although in several $\operatorname{articles}^{13}$ the Mayfield frame was placed on the prone patient and attached to the Jackson table, we found it works just as well on the typical neurosurgery operating table (for example, Skytron) as long as the Mayfield support/ mounting device is reversed so as to have a low profile to fit into the donut of the O-arm (Fig. 11A). Removal of the tabletop cushion above the thoracic chest, as well as using small thoracic bolsters, reduces the height.

Limiting intersegmental movement of the vertebrae by placing the retractors prior to the intraoperative $\mathrm{CT}$ was also very important. Of note, the C-1 vertebra is quite mobile, and C-1 screws should not be advanced while following the navigation. The trajectory should be used and the screws advanced in small steps; navigation should be used when the small screw advancement is temporarily stopped. We have found this to be a problem mainly at C-1.

Finally, we must remember that CT image guidance should be used as an aid in spine surgery. It does not replace anatomical knowledge, which should always be used, if possible, when placing hardware. We have also found that frequent checking of familiar landmarks keeps the surgeon aware of any changes in the accuracy of the guidance system.

\section{Conclusions}

There is no doubt that with the advancement of image guidance the challenges of spine surgery at the occipitocervical junction have been reduced. The 4 cases described in this paper illustrate not only the accuracy of placement of instrumentation in this region with the $\mathrm{O}$-arm surgical imaging system and StealthStation navigation, but also use of this system in decompression of complex anatomy involving spine deformity, en bloc resection, and revision surgery with the added value of being able to evaluate the bony decompression and instrumentation before the patient leaves the operating room.

\section{Disclosure}

The authors report no conflict of interest concerning the materials or methods used in this study or the findings specified in this paper.

Author contributions to the study and manuscript preparation include the following. Conception and design: Guppy. Acquisition of data: Guppy, Chakrabarti. Analysis and interpretation of data: Guppy. Drafting the article: all authors. Critically revising the article: all authors. Reviewed submitted version of manuscript: all authors. Approved the final version of the manuscript on behalf of all authors: Guppy. Administrative/technical/material support: Guppy. Study supervision: Guppy.

\section{References}

1. Baaj AA, Beckman J, Smith DA: O-Arm-based image guidance in minimally invasive spine surgery: technical note. Clin Neurol Neurosurg 115:342-345, 2013

2. Costa F, Cardia A, Ortolina A, Fabio G, Zerbi A, Fornari M: Spinal navigation: standard preoperative versus intraoperative computed tomography data set acquisition for computer-guidance system: radiological and clinical study in 100 consecutive patients. Spine (Phila Pa 1976) 36:2094-2098, 2011

3. Del Curto D, Kim JS, Lee SH: Minimally invasive posterior cervical microforaminotomy in the lower cervical spine and $\mathrm{C}-\mathrm{T}$ junction assisted by $\mathrm{O}$-arm-based navigation. Comput Aided Surg 18:76-83, 2013

4. Drazin D, Liu JC, Acosta FL Jr: CT navigated lateral interbody fusion. J Clin Neurosci 20:1438-1441, 2013

5. Gebhard FT, Kraus MD, Schneider E, Liener UC, Kinzl L, Arand M: Does computer-assisted spine surgery reduce intraoperative radiation doses? Spine (Phila Pa 1976) 31:20242028, 2006

6. Guppy KH, Chakrabarti I, Isaacs RS, Jun JH: En bloc resection of a multilevel high-cervical chordoma involving C-2: new operative modalities. Technical note. J Neurosurg Spine 19:232-242, 2013

7. Holly LT, Foley KT: Intraoperative spinal navigation. Spine (Phila Pa 1976) 28 (15 Suppl):S54-S61, 2003

8. Hsu W, Kosztowski TA, Zaidi HA, Gokaslan ZL, Wolinsky JP: Image-guided, endoscopic, transcervical resection of cervical chordoma. Technical note. J Neurosurg Spine 12:431435,2010

9. Ishikawa Y, Kanemura T, Yoshida G, Matsumoto A, Ito Z, Tauchi R, et al: Intraoperative, full-rotation, three-dimensional image (O-arm)-based navigation system for cervical pedicle screw insertion. Clinical article, J Neurosurg Spine 15: 472-478, 2011

10. Li L, Wang P, Chen L, Ma X, Bu B, Yu X: Individualized treatment of craniovertebral junction malformation guided by intraoperative computed tomography. J Spinal Disord Tech 25:77-84, 2012

11. Mattei TA, Fassett DR: The O-arm revolution in spine surgery. J Neurosurg Spine 19:644-647, 2013 (Letter)

12. Mummaneni PV, Haid RW: Transoral odontoidectomy. Neurosurgery 56:1045-1050, 2005

13. Nottmeier EW, Pirris SM: Placement of thoracic transvertebral pedicle screws using 3D image guidance. Technical note. J Neurosurg Spine 18:479-483, 2013

14. Nottmeier EW, Pirris SM, Edwards S, Kimes S, Bowman C, Nelson KL: Operating room radiation exposure in cone beam computed tomography-based, image-guided spinal surgery. Clinical article. J Neurosurg Spine 19:226-231, 2013

15. Nottmeier EW, Young PM: Image-guided placement of occipitocervical instrumentation using a reference arc attached to the headholder. Neurosurgery 66 (3 Suppl Operative): 138-142, 2010

16. Ray WZ, Ravindra VM, Schmidt MH, Dailey AT: Stereotactic navigation with the $\mathrm{O}$-arm for placement of S-2 alar iliac screws in pelvic lumbar fixation. Technical note. J Neurosurg Spine 18:490-495, 2013

17. Scheufler KM, Franke J, Eckardt A, Dohmen H: Accuracy of image-guided pedicle screw placement using intraoperative computed tomography-based navigation with automated referencing, part I: cervicothoracic spine. Neurosurgery 69: 782-795, 2011

18. Schouten R, Lee R, Boyd M, Paquette S, Dvorak M, Kwon BK, et al: Intra-operative cone-beam CT (O-arm) and stereotactic navigation in acute spinal trauma surgery. J Clin Neurosci 19:1137-1143, 2012

19. Seichi A, Takeshita K, Nakajima S, Akune T, Kawaguchi H, Nakamura K: Revision cervical spine surgery using transarticular or pedicle screws under a computer-assisted imageguidance system. J Orthop Sci 10:385-390, 2005

20. Shin MH, Hur JW, Ryu KS, Park CK: Prospective comparison study between the fluoroscopy guided and navigation coupled with $\mathrm{O}$-arm ${ }^{\circledR}$ guided pedicle screw placement in the thoracic and lumbosacral spines. J Spinal Disord Tech [epub ahead of print], 2013 


\section{K. H. Guppy, I. Chakrabarti, and A. Banerjee}

21. Silbermann J, Riese F, Allam Y, Reichert T, Koeppert H, Gutberlet M: Computer tomography assessment of pedicle screw placement in lumbar and sacral spine: comparison between free-hand and O-arm based navigation techniques. Eur Spine J 20:875-881, 2011

22. Tormenti MJ, Kostov DB, Gardner PA, Kanter AS, Spiro RM, Okonkwo DO: Intraoperative computed tomography imageguided navigation for posterior thoracolumbar spinal instrumentation in spinal deformity surgery. Neurosurg Focus 28(3): E11, 2010

23. Van de Kelft E, Costa F, Van der Planken D, Schils F: A prospective multicenter registry on the accuracy of pedicle screw placement in the thoracic, lumbar, and sacral levels with the use of the O-arm imaging system and StealthStation Navigation. Spine (Phila Pa 1976) 37:E1580-E1587, 2012

24. Veres R, Bagó A, Fedorcsák I: Early experiences with imageguided transoral surgery for the pathologies of the upper cervical spine. Spine (Phila Pa 1976) 26:1385-1388, 2001
25. Yu X, Li L, Wang P, Yin Y, Bu B, Zhou D: Intraoperative computed tomography with an integrated navigation system in stabilization surgery for complex craniovertebral junction malformation. J Spinal Disord Tech [epub ahead of print], 2012

Manuscript submitted November 14, 2013.

Accepted January 9, 2014.

Please include this information when citing this paper: DOI: 10.3171/2014.1.FOCUS13514.

Address correspondence to: Kern H. Guppy, M.D., Ph.D., The Kaiser Permanente Medical Group, Sacramento, and Department of Neurosurgery, University of California, San Francisco, California, 2025 Morse Ave., Sacramento, CA 95825-2115. email: kguppy@ yahoo.com. 